\title{
Benchmarking the NEWUOA on the BBOB-2009 Function Testbed
}

\author{
Raymond Ros \\ Univ. Paris-Sud, LRI \\ UMR 8623 / INRIA Saclay, projet TAO \\ F-91405 Orsay, France \\ raymond.ros@Iri.fr
}

\begin{abstract}
The NEWUOA which belongs to the class of Derivative-Free optimization algorithms is benchmarked on the BBOB-2009 noisefree testbed. A multistart strategy is applied with a maximum number of function evaluations of up to $10^{5}$ times the search space dimension resulting in the algorithm solving 11 functions in 20-D. The results of the algorithm using the recommended number of interpolation points for the underlying model and the full model are shown and discussed.
\end{abstract}

\section{Categories and Subject Descriptors}

G.1.6 [Numerical Analysis]: Optimization-global optimization, unconstrained optimization; F.2.1 [Analysis of Algorithms and Problem Complexity]: Numerical Algorithms and Problems

\section{General Terms}

Algorithms

\section{Keywords}

Benchmarking, Black-box optimization, Derivative-free optimization

\section{ALGORITHM PRESENTATION}

The NEWUOA (New Unconstrained Optimization Algorithm) [4] is a Derivative-Free Optimization (DFO) algorithm using the trust region paradigm. NEWUOA computes a quadratic interpolation of the objective function in the current trust region and performs a truncated conjugate gradient minimization of the surrogate model in the trust region. It then updates either the current best point or the radius of the trust region, based on the a posteriori interpolation error. The time complexity of the algorithm is $\mathcal{O}\left(m^{2} n\right)$ in the worst case but in practice closer to $\mathcal{O}(m n)$, where $m$ is the number of interpolation points used for the determination of the quadratic model and $n$ is the dimension

Permission to make digital or hard copies of all or part of this work for personal or classroom use is granted without fee provided that copies are not made or distributed for profit or commercial advantage and that copies bear this notice and the full citation on the first page. To copy otherwise, to republish, to post on servers or to redistribute to lists, requires prior specific permission and/or a fee.

GECCO'09, July 8-12, 2009, Montréal Québec, Canada.

Copyright 2009 ACM 978-1-60558-505-5/09/07 ...\$5.00. of the search space. The number of interpolation points is a parameter of the algorithm and needs to be chosen in the range $\left[n+2, \frac{(n+1)(n+2)}{2}\right]$. Other parameters of the algorithm are the initial and final radii of the trust region, respectively governing the initial 'granularity' and the precision of the search. A simple stochastic independent restart procedure (as advised in [2]) was added to improve the probability of the algorithm reaching a target function value.

\section{EXPERIMENTAL PROCEDURE}

The implementation used for our experiments is the one provided by Matthieu Guibert ${ }^{1}$ which delivers Powell's original Fortran source code of the algorithm. This Fortran code has been adapted to the BBOB experimental paradigm. In this paper, we will test two numbers of interpolation points: $2 n+1$ which is recommended in [4] and $\frac{(n+1)(n+2)}{2}$ which is the full model. An intermediate model using a number of interpolation points that is the integer closer to $\sqrt{(n+1 / 2)(n+1)(n+2)}$ was also tested with results that were in-between those of the two models we are considering. The initial radius $\rho_{\text {beg }}$ of the search region has been set to 10 , the range of the search space. Preliminary experiments shows very few dependencies on this parameter, given it is not too small (ie. by many orders of magnitude) for the problem considered. A final radius $\rho_{\text {end }}=10^{-16}$ was chosen close to the limit being the machine precision to prevent numerical errors. The starting point $x_{0}$ is chosen uniformly in $[-5,5]^{n}$. The multistart strategy was used with at most 100 restarts to reduce the duration of an experiment. For the same reason, the maximum number of function evaluations is $10^{5} \times n$ for $m=2 n+1,10^{4} \times n$ otherwise. An example of the algorithm used is presented in Figure 1. No parameter tuning was done, the CrE [2] is computed to zero.

\section{RESULTS AND DISCUSSION}

Results from experiments according to [2] on the benchmark functions given in [1,3] are presented in Figures 2 and 3 and in Table 1 for $m=2 n+1$. The algorithm performs well on the convex quadratic functions $f_{1}$. It solves $f_{2}$ and $f_{11}$. The algorithm performs well on functions with low or moderate conditioning.

On multimodal functions, the algorithm fails or only solves 2, 3 and/or 5-D, though it does well on the Gallagher functions. As we can see in Figures 4 and 5 and in Table 2 for the full model, these results cannot be improved by using more ${ }^{1}$ http://www.inrialpes.fr/bipop/people/guilbert/
newuoa/newuoa.html 

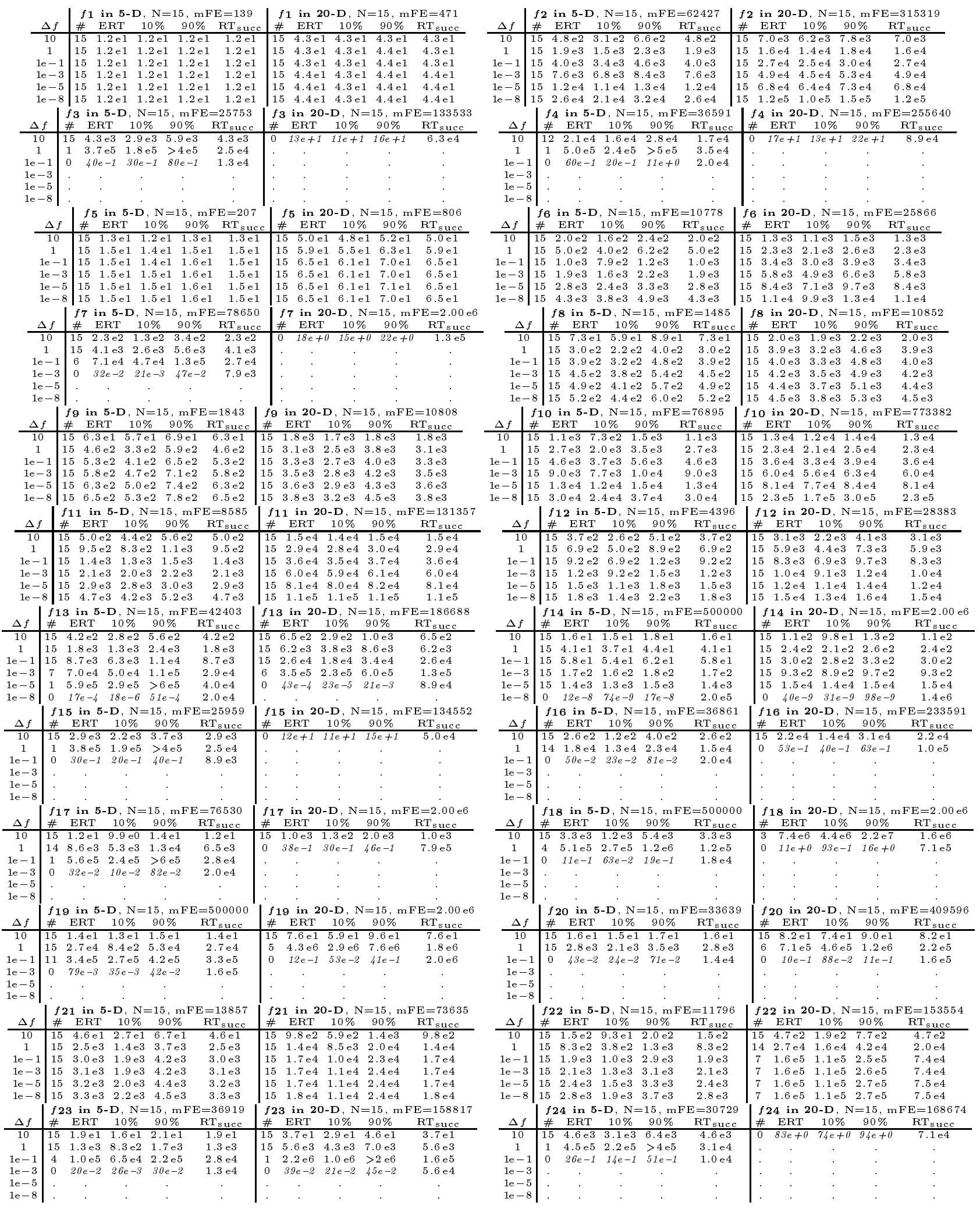

Table 1: NEWUOA, $2 n+1$ interpolation points. Shown are, for a given target difference to the optimal function value $\Delta f$ : the number of successful trials (\#); the expected running time to surpass $f_{\mathrm{opt}}+\Delta f$ (ERT, see Figure 2); the 10\%-tile and $90 \%$-tile of the bootstrap distribution of ERT; the average number of function evaluations in successful trials or, if none was successful, as last entry the median number of function evaluations to reach the best function value $\left(\mathbf{R T}_{\text {succ }}\right)$. If $f_{\mathrm{opt}}+\Delta f$ was never reached, figures in italics denote the best achieved $\Delta f$-value of the median trial and the $10 \%$ and $90 \%$-tile trial. Furthermore, $N$ denotes the number of trials, and mFE denotes the maximum of number of function evaluations executed in one trial. See Figure 2 for the names of functions. 



Table 2: NEWUOA, full model. Shown are, for a given target difference to the optimal function value $\Delta f$ : the number of successful trials (\#); the expected running time to surpass $f_{\mathrm{opt}}+\Delta f$ (ERT, see Figure 2); the $10 \%$-tile and $90 \%$-tile of the bootstrap distribution of ERT; the average number of function evaluations in successful trials or, if none was successful, as last entry the median number of function evaluations to reach the best function value $\left(\mathbf{R T}_{\text {succ }}\right)$. If $f_{\mathrm{opt}}+\Delta f$ was never reached, figures in italics denote the best achieved $\Delta f$-value of the median trial and the $10 \%$ and $90 \%$-tile trial. Furthermore, $\mathrm{N}$ denotes the number of trials, and $\mathrm{mFE}$ denotes the maximum of number of function evaluations executed in one trial. See Figure 2 for the names of functions. 
Figure 1: Multistart NEWUOA, the number of interpolation points is two times the dimension plus one.

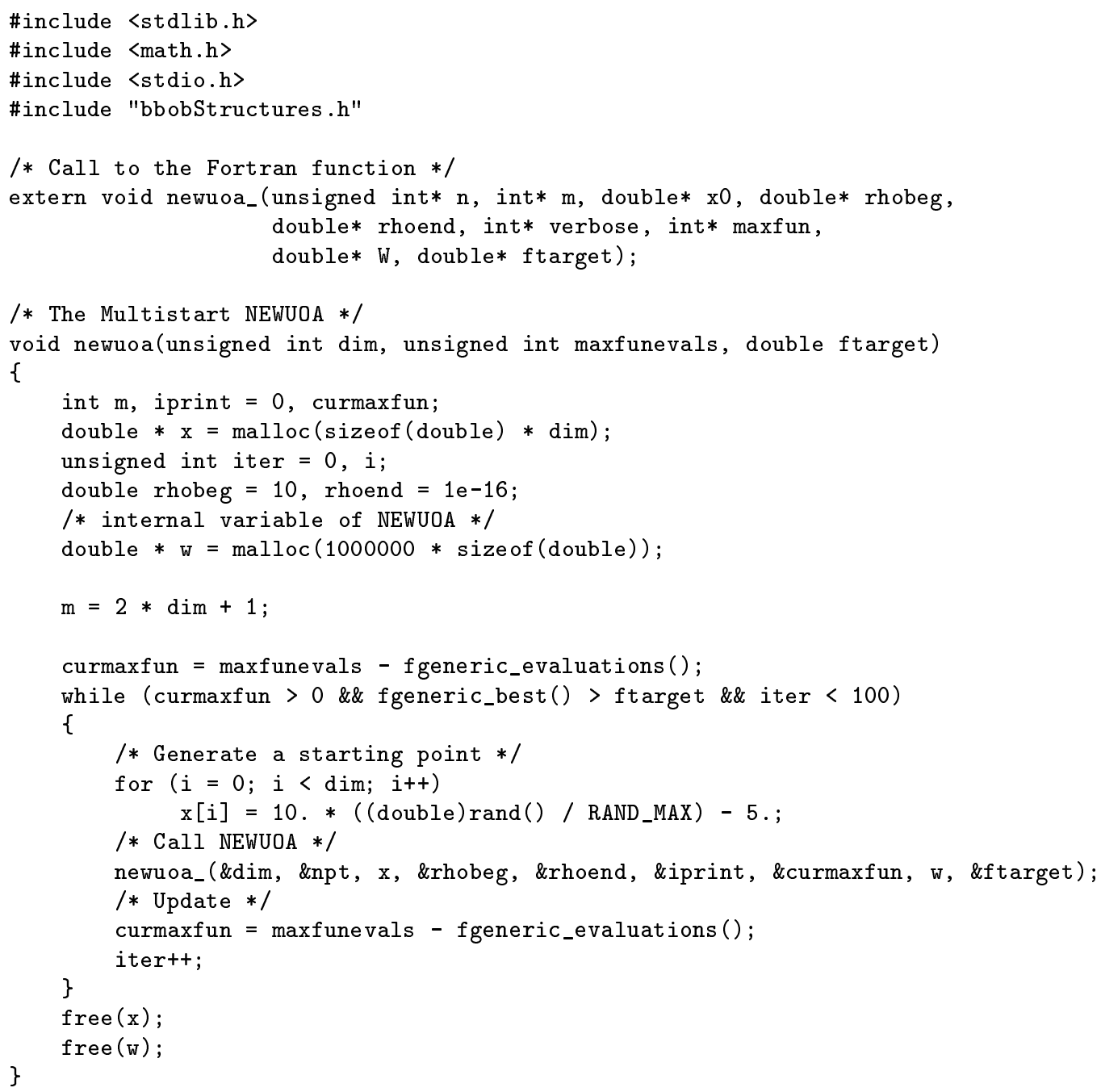

points on the interpolation of the model. To the contrary, the performances only seem to scale only worse resulting in failures in larger dimensions, for instance on $f_{2}$ or $f_{12}$ with the exception of $f_{7}$ which the full model NEWUOA solves in 5 -D.

\section{CPU TIMING EXPERIMENT}

The proposed algorithm was run on $f_{8}$ and restarted until at least 30 seconds have passed. The experiments were conducted with an Intel Core 26700 processor $(2.66 \mathrm{GHz})$ on Linux 2.6.24.7. The results were 130, 73, 45, 18, 2.2, 26 for $m=2 n+1$ and $200,86,45,7.9,3.7,36 \times 10^{-3}$ seconds per function evaluations for the full model in dimension 2 , $3,5,10,20$ and 40 respectively.

\section{Acknowledgments}

The first author would like to acknowledge the support, help, and work of the BBOB team with particular kudos to Anne Auger, Steffen Finck and Nikolaus Hansen.

\section{REFERENCES}

[1] S. Finck, N. Hansen, R. Ros, and A. Auger. Real-parameter black-box optimization benchmarking 2009: Presentation of the noiseless functions. Technical Report 2009/20, Research Center PPE, 2009.

[2] N. Hansen, A. Auger, S. Finck, and R. Ros. Real-parameter black-box optimization benchmarking 2009: Experimental setup. Technical Report RR-6828, INRIA, 2009.

[3] N. Hansen, S. Finck, R. Ros, and A. Auger. Real-parameter black-box optimization benchmarking 2009: Noiseless functions definitions. Technical Report RR-6829, INRIA, 2009.

[4] M. J. D. Powell. The NEWUOA software for unconstrained optimization without derivatives. Large Scale Nonlinear Optimization, pages 255-297, 2006. 

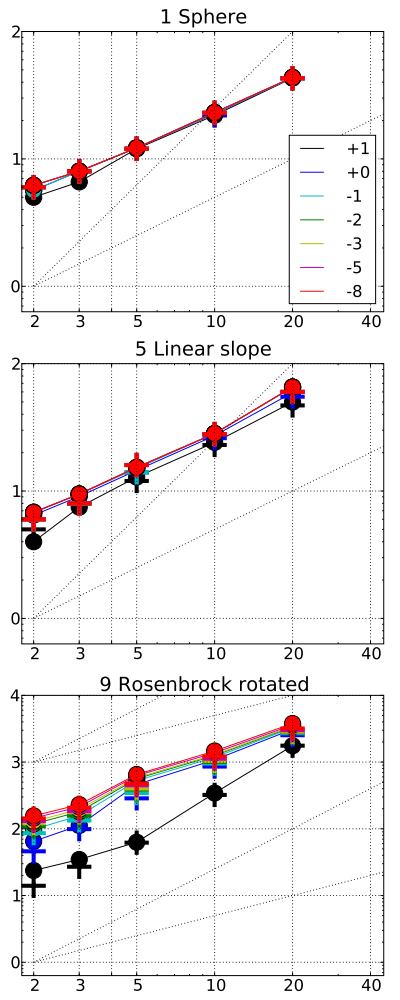

13 Sharp ridge
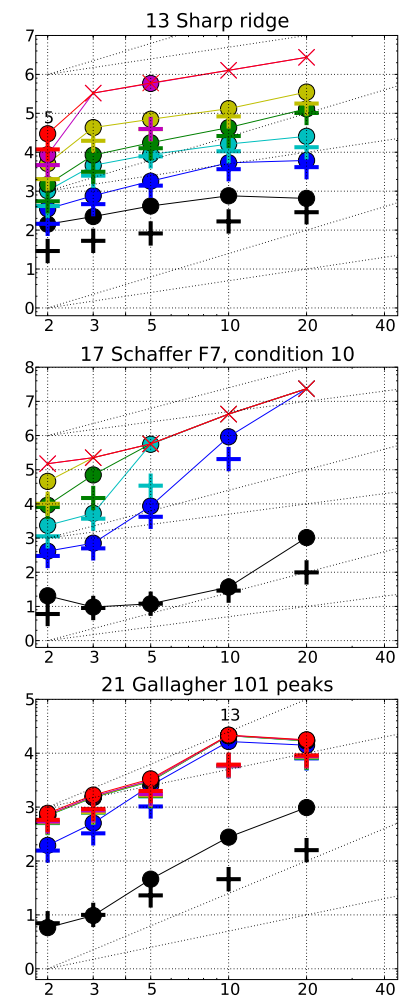
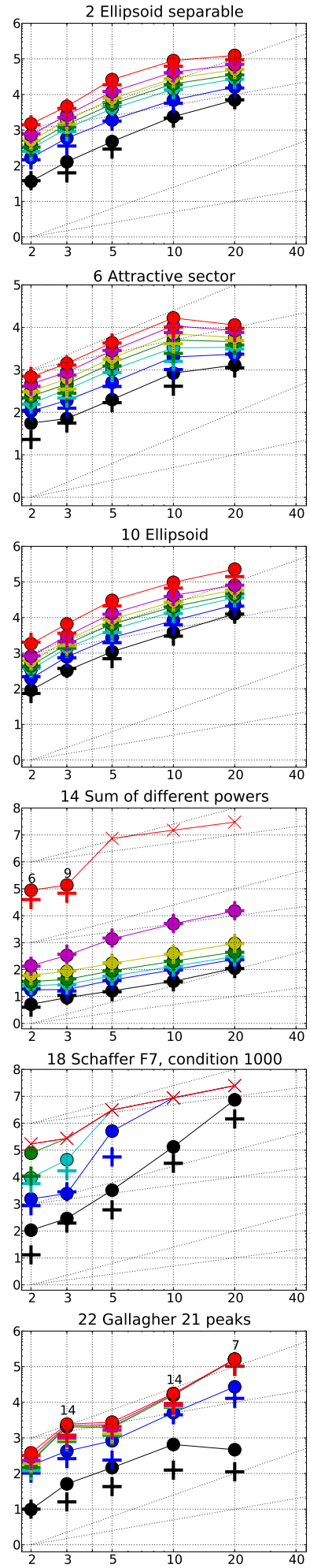
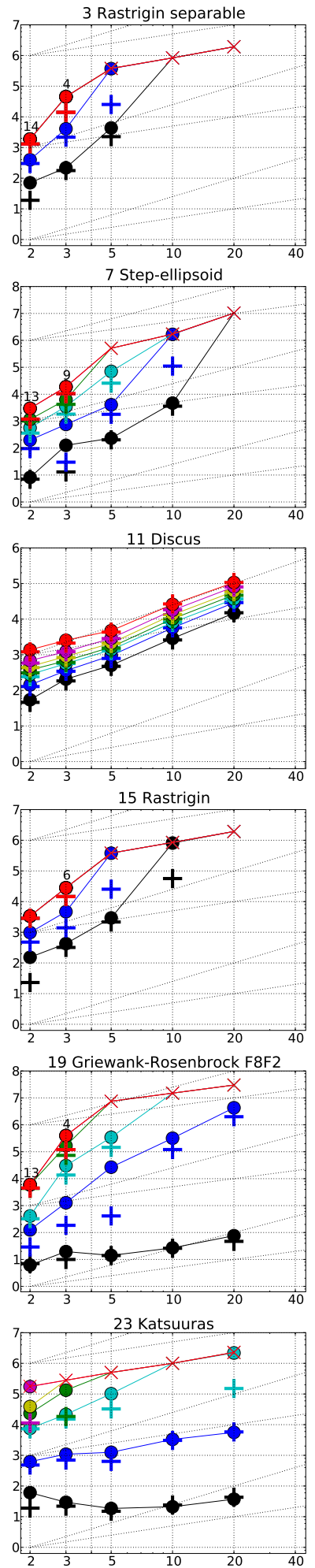
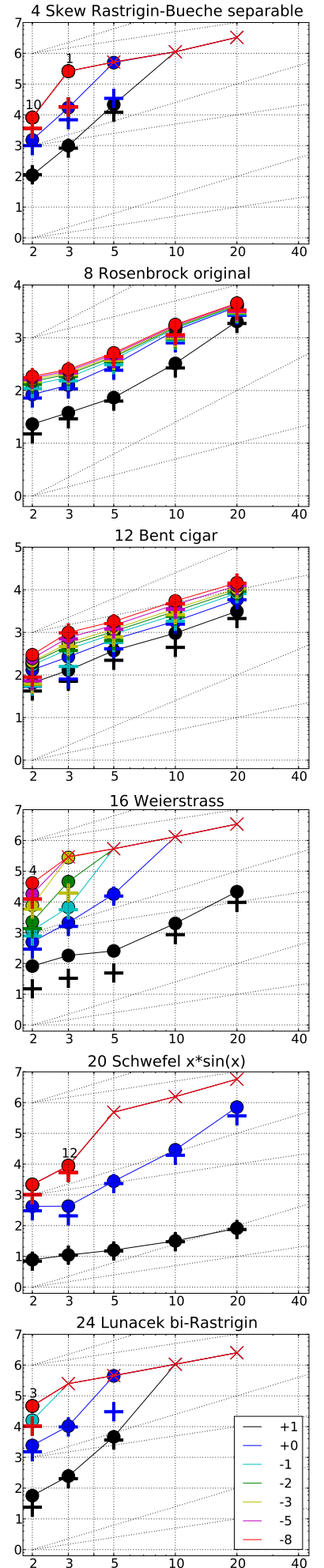

Figure 2: NEWUOA, $2 n+1$ interpolation points. Expected Running Time (ERT, •) to reach $f_{\mathrm{opt}}+\Delta f$ and median number of function evaluations of successful trials $(+)$, shown for $\Delta f=10,1,10^{-1}, 10^{-2}, 10^{-3}, 10^{-5}, 10^{-8}$ (the exponent is given in the legend of $f_{1}$ and $\left.f_{24}\right)$ versus dimension in $\log$ - $\log$ presentation. The ERT $(\Delta f)$ equals to \#FEs $(\Delta f)$ divided by the number of successful trials, where a trial is successful if $f_{\mathrm{opt}}+\Delta f$ was surpassed during the trial. The $\# \operatorname{FEs}(\Delta f)$ are the total number of function evaluations while $f_{\text {opt }}+\Delta f$ was not surpassed during the trial from all respective trials (successful and unsuccessful), and $f_{\mathrm{opt}}$ denotes the optimal function value. Crosses $(x)$ indicate the total number of function evaluations \#FEs $(-\infty)$. Numbers above ERT-symbols indicate the number of successful trials. Annotated numbers on the ordinate are decimal logarithms. Additional grid lines show linear and quadratic scaling. 
$D=5$
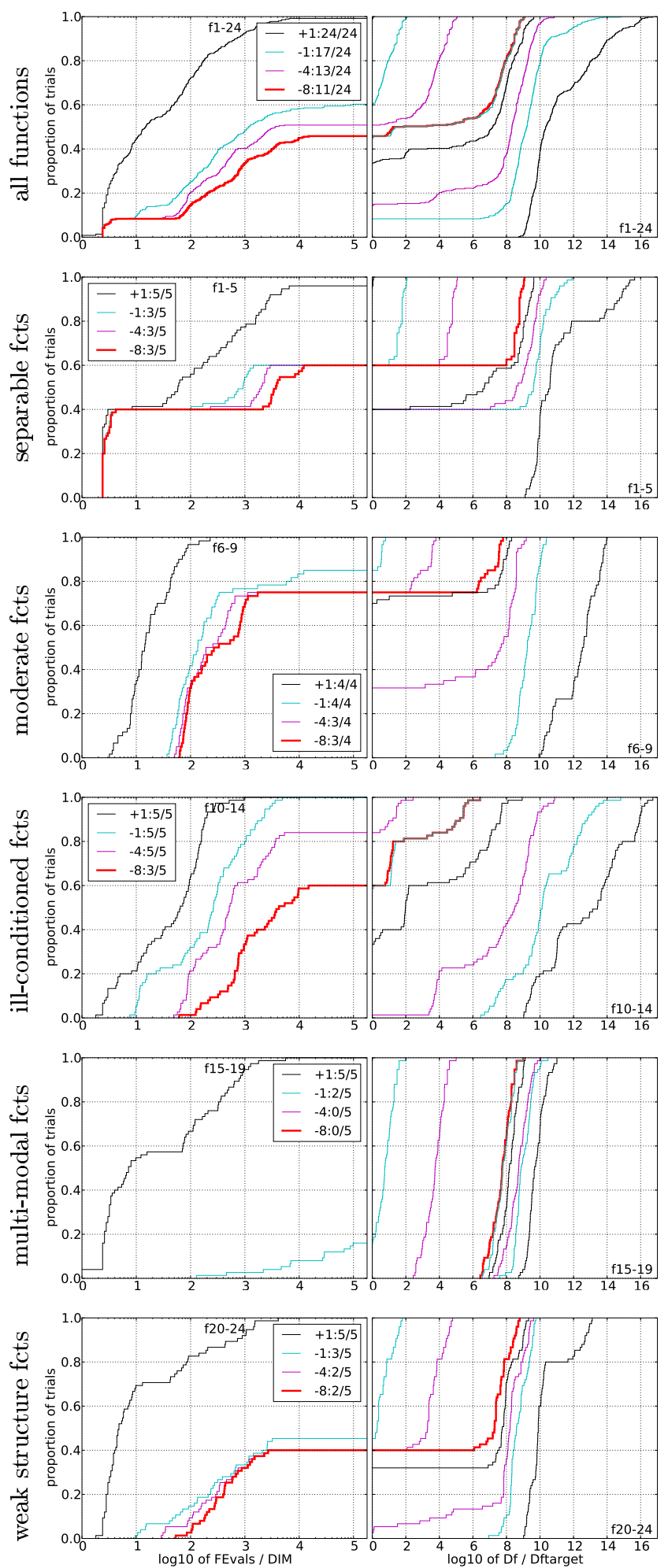

$D=20$
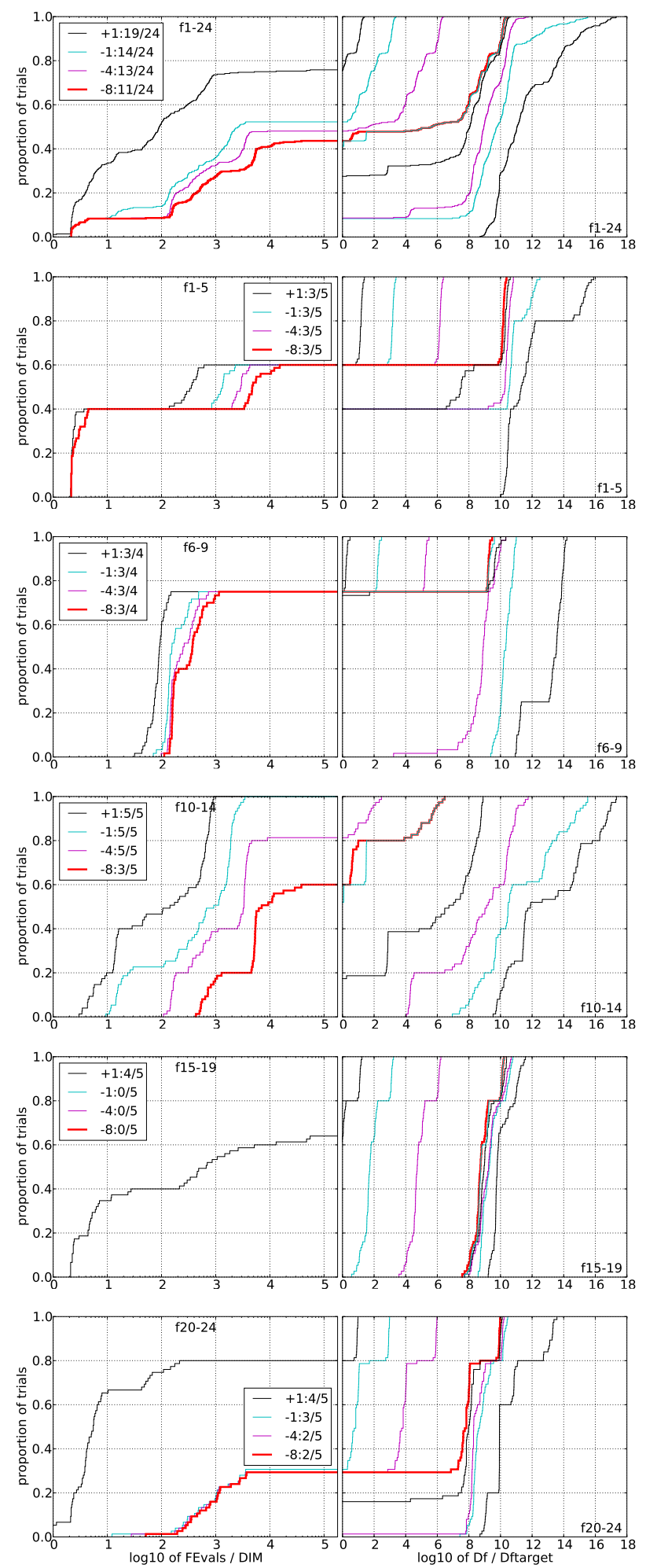

Figure 3: NEWUOA, $2 n+1$ interpolation points. Empirical cumulative distribution functions (ECDFs), plotting the fraction of trials versus running time (left subplots) or versus $\Delta f$ (right subplots). The thick red line represents the best achieved results. Left subplots: ECDF of the running time (number of function evaluations), divided by search space dimension $D$, to fall below $f_{\text {opt }}+\Delta f$ with $\Delta f=10^{k}$, where $k$ is the first value in the legend. Right subplots: ECDF of the best achieved $\Delta f$ divided by $10^{k}$ (upper left lines in continuation of the left subplot), and best achieved $\Delta f$ divided by $10^{-8}$ for running times of $D, 10 D, 100 D \ldots$ function evaluations (from right to left cycling black-cyan-magenta). Top row: all results from all functions; second row: separable functions; third row: misc. moderate functions; fourth row: ill-conditioned functions; fifth row: multi-modal functions with adequate structure; last row: multi-modal functions with weak structure. The legends indicate the number of functions that were solved in at least one trial. FEvals denotes number of function evaluations, $D$ and DIM denote search space dimension, and $\Delta f$ and Df denote the difference to the optimal function value. 

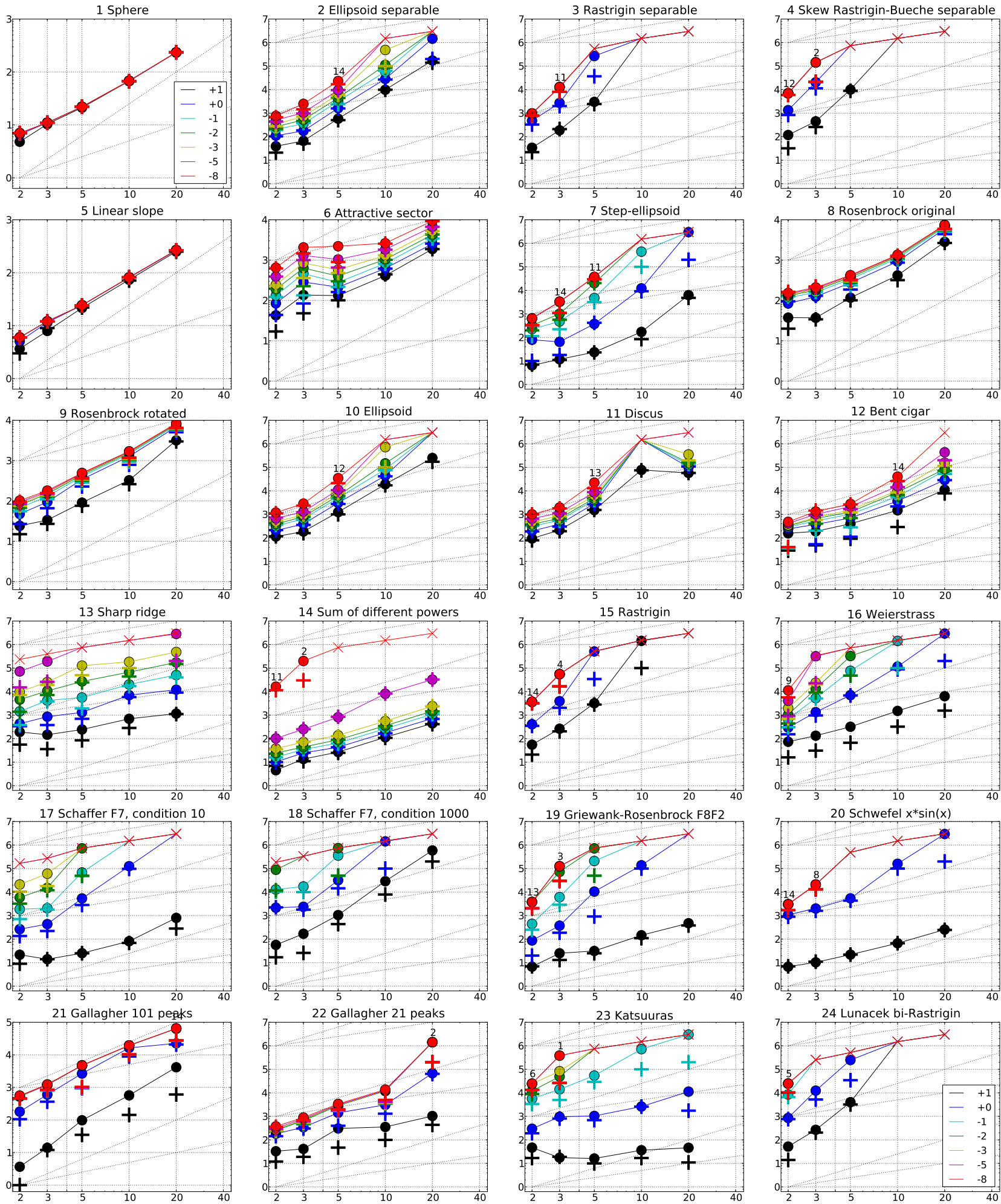

Figure 4: NEWUOA, full model. Expected Running Time (ERT, •) to reach $f_{\text {opt }}+\Delta f$ and median number of function evaluations of successful trials $(+)$, shown for $\Delta f=10,1,10^{-1}, 10^{-2}, 10^{-3}, 10^{-5}, 10^{-8}$ (the exponent is given in the legend of $f_{1}$ and $\left.f_{24}\right)$ versus dimension in log-log presentation. The $\operatorname{ERT}(\Delta f)$ equals to \#FEs $(\Delta f)$ divided by the number of successful trials, where a trial is successful if $f_{\text {opt }}+\Delta f$ was surpassed during the trial. The \#FEs $(\Delta f)$ are the total number of function evaluations while $f_{\mathrm{opt}}+\Delta f$ was not surpassed during the trial from all respective trials (successful and unsuccessful), and $f_{\text {opt }}$ denotes the optimal function value. Crosses $(x)$ indicate the total number of function evaluations \#FEs $(-\infty)$. Numbers above ERT-symbols indicate the number of successful trials. Annotated numbers on the ordinate are decimal logarithms. Additional grid lines show linear and quadratic scaling. 
$D=5$
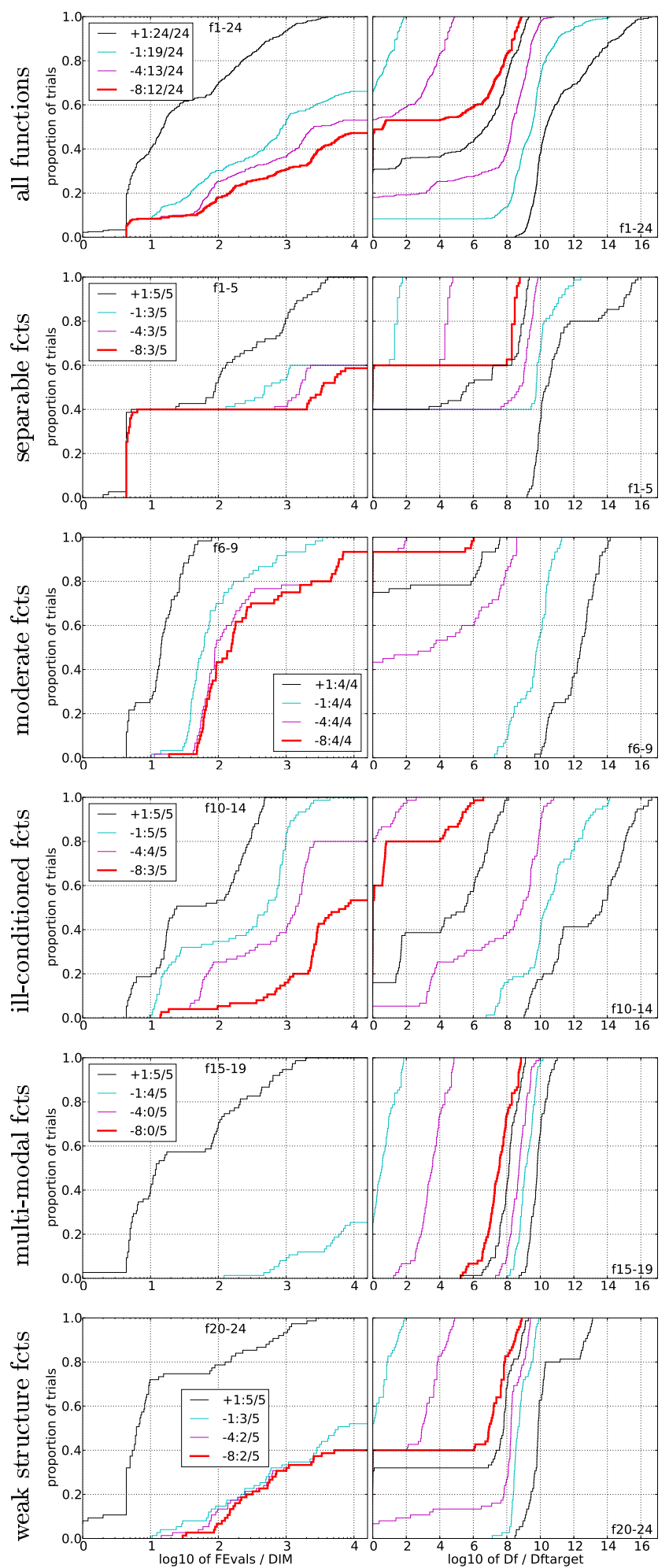

$D=20$
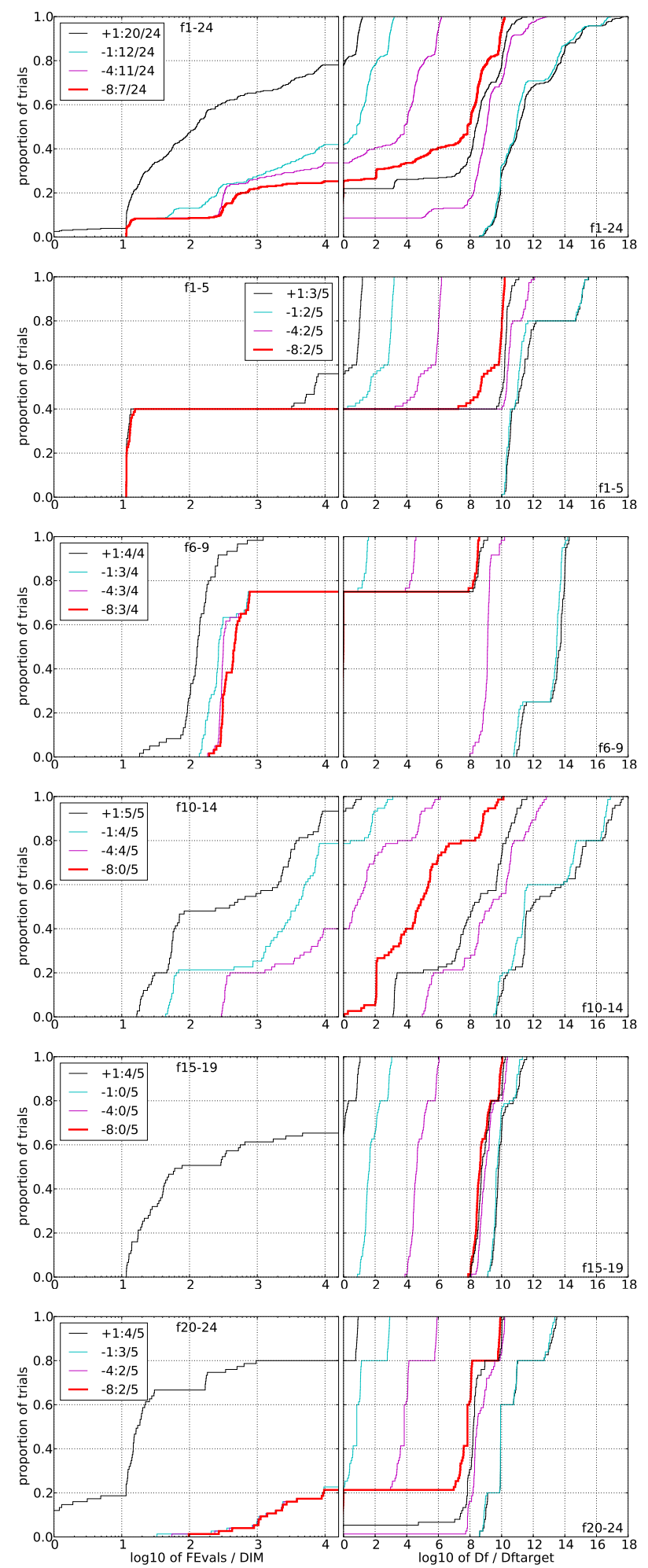

Figure 5: NEWUOA, full model. Empirical cumulative distribution functions (ECDFs), plotting the fraction of trials versus running time (left subplots) or versus $\Delta f$ (right subplots). The thick red line represents the best achieved results. Left subplots: ECDF of the running time (number of function evaluations), divided by search space dimension $D$, to fall below $f_{\mathrm{opt}}+\Delta f$ with $\Delta f=10^{k}$, where $k$ is the first value in the legend. Right subplots: ECDF of the best achieved $\Delta f$ divided by $10^{k}$ (upper left lines in continuation of the left subplot), and best achieved $\Delta f$ divided by $10^{-8}$ for running times of $D, 10 D, 100 D \ldots$ function evaluations (from right to left cycling black-cyan-magenta). Top row: all results from all functions; second row: separable functions; third row: misc. moderate functions; fourth row: ill-conditioned functions; fifth row: multi-modal functions with adequate structure; last row: multi-modal functions with weak structure. The legends indicate the number of functions that were solved in at least one trial. FEvals denotes number of function evaluations, $D$ and DIM denote search space dimension, and $\Delta f$ and Df denote the difference to the optimal function value. 